\title{
О ВЛИЯНИИ ПРОЦЕССОВ ИСПАРЕНИЯ ВОДЫ НА ЭФФЕКТИВНОСТЬ ОХЛАЖДЕНИЯ В ГРАДИРНЯХ
}

\author{
Шевелев Сергей Анатольевич', \\ wevelev@tpu.ru \\ Зяблова Наталия Николаевна', \\ zyablova@tpu.ru \\ 1 Национальный исследовательский Томский политехнический университет, \\ Россия, 634050, г. Томск, пр. Ленина, 30.
}

Представлены результаты теоретического исследования охлаждения капель воды при их движении в потоке холодного воздуха в условиях, характерных для башенных градирен большой производительности, и результаты численного анализа распределений температуры по радиусу капель. Вследствие больших перепадов температур и значительных скоростей движения в рассматриваемой системе распределение температур внутри капель принято изменяющимся по радиусу. Учтено повышение влажности воздуха в пристеночной области за счет испарения воды. Полученная система нестационарных дифференциальных уравнений в частных производных решена методом конечных разностей с использованием неявной четырехточечной разностной схемы. Решение задачи с подвижной границей проведено методом ловли фронта в узел пространственной сетки. Для решения нелинейных уравнений применялся метод итераций. Цель работы - численная оценка вклада процессов конвективного и испарительного охлаждения в формирование температурного поля капли воды в условиях, типичных для современных градирен. Анализ проведен для двух характерных значений температуры окружающего воздуха, соответствующих летнему и зимнему периодам года. Выполнен анализ влияния охлаждения капель за счет испарения воды и теплопроводности. Получены распределения температуры по радиусу для различных значений характерных размеров капель. Показано, что изменение радиуса для всего диапазона характерных размеров капель за время их полета составляет менее 0,73 \% и не оказывает значительного влияния на величину охлаждения воды в градирне. Установлено, что поглощение теплоты испарением играет важную роль в формировании распределения температур в каплях оборотной воды на выходе из градирен. Показано, что при отрицательных температурах наружного воздуха чрезмерное дробление капель является нецелесообразным.

\section{Ключевые слова:}

Оборотная вода, тепломассоперенос, градирня, испарение воды, конвекция.

\section{Введение}

Оборотная вода, используемая во многих технологических процессах различных отраслей промышленности (химическая [1, 2], нефтехимическая [3], энергетика [4-6]), как правило, охлаждается в градирнях при движении капель через поток воздуха, температура которого существенно меньше (на $30-60{ }^{\circ} \mathrm{C}$ ) температуры воды. Такие системы охлаждения достаточно часто называются испарительными [2, 3], но пока нет конкретных данных о том, какой вклад в процесс снижения температуры капель воды вносит конвекция, а какой испарение. Выполнить экспериментальные оценки интенсивности каждого из этих двух процессов невозможно, потому что нельзя в практически значимых условиях теплообмена капель воды с потоком относительно холодного воздуха разделить охлаждение в процессе конвективного теплоотвода и за счет испарения воды. Решение такой задачи возможно только при математическом моделировании процессов тепло- и массопереноса при движении капель нагретой относительно внешней среды воды через встречный поток холодного воздуха.

Цель работы - численная оценка вклада процессов конвективного и испарительного охлаждения в формирование температурного поля капли воды в условиях, типичных для современных градирен.

\section{Постановка задачи и метод решения}

При решении многих задач, в которых необходим анализ температуры капель жидкостей (в том числе и воды), принимается допущение $[7,8]$ об однородности поля температур капли в любых условиях ее нагрева или охлаждения. Но охлаждение капель воды в градирнях происходит, как правило, при интенсивном отводе теплоты от капель (достаточно большие перепады температур в системе «капля воды - воздух" и скорости движения воздуха). Поэтому, учитывая низкую теплопроводность воды при умеренных температурах (менее $60{ }^{\circ} \mathrm{C}$ ), можно предположить, что в типичных условиях работы градирен возможно возникновение больших градиентов температуры в приповерхностных слоях капель воды. Следовательно, для адекватного описания температурного распределения в каплях (скорее всего, существенно нестационарного) необходимо решение дифференциального уравнения теплопроводности в частных производных. При изучении влияния процессов фазовых превращений при постановке задачи необходимо учитывать, как показывает анализ литературы по проблеме испарения капель $[9,10]$, два фактора, наиболее существенно влияющих на скорость этого процесса: температуру поверхности испарения и парциальное давление паров вблизи этой поверхности.

По результатам экспериментальных исследований $[11,12]$ сделан вывод, что при движении в по- 
токе газов (независимо от их температуры) капли всех жидкостей непрерывно деформируются. Решение задачи трансформации капли в полете под действием сил тяжести представляет собой самостоятельную сложную проблему. Кроме того, peшение задачи для тел неправильной формы также очень сложно. Поэтому при постановке задачи принято допущение, что капля воды сохраняет в полете форму сферы. Можно отметить, что при решении задачи тепломассопереноса в каплях, как правило, предполагается, что последние имеют форму сферы.

\section{Физическая постановка задачи}

Рассматривалась капля воды, движущаяся во встречном потоке холодного воздуха. Проанализированы два варианта постановки задачи.

В первом варианте предполагалось, что испарение не оказывает заметного влияния на распределение температур внутри капли, а ее охлаждение происходит только за счет конвективного теплообмена. Так как парообразование на поверхности капли в рассматриваемой постановке не учитывалось, то теплофизические характеристики окружающего каплю воздуха сохранялись неизменными.

Во втором варианте постановки задачи принято, что охлаждение происходит при непосредственном контакте нагретой капли воды с потоком относительно холодного воздуха. При этом отвод теплоты от капли происходит за счет конвективного теплообмена и поглощения теплоты парообразования. В результате испарения образуется водяной пар, который вдувается в малую область вблизи стенки капли, где смешивается с воздухом. Это приводит к повышению влажности воздуха в пристеночной области. Движение капли происходит в образовавшейся парогазовой среде.

Теплообмен излучением в условиях градирен мал и не учитывался ни в одной из рассмотренных постановок.

\section{Математическая постановка задачи}

Схема области решения для первого варианта постановки задачи представлена на рис. 1.

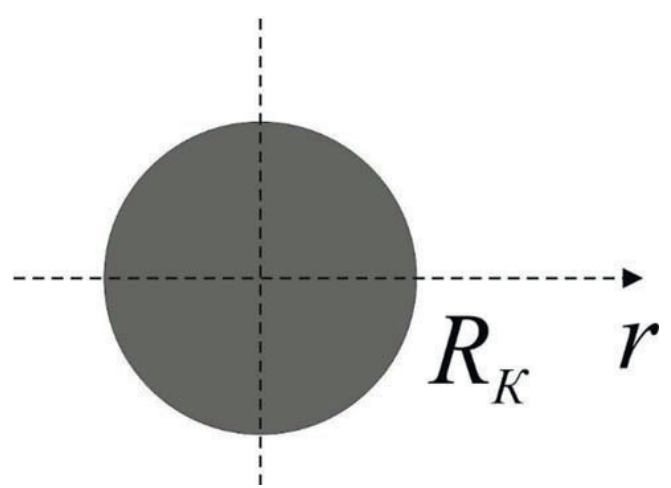

Рис. 1. Схема области решения для первого варианта постанов ки задачи

Fig. 1. Scheme of solution region for the first variant of the problem statement
Распределение температуры в капле описывается уравнением теплопроводности:

$$
\rho c \frac{\partial T}{\partial t}=\lambda\left(\frac{\partial T^{2}}{\partial r^{2}}+\frac{2}{r} \frac{\partial T}{\partial r}\right), 0<r<R_{\mathrm{K}}
$$

где $\rho$ - плотность; $c$ - теплоемкость; $T$ - температура воды; $r$ - радиальная координата; $R_{\kappa}$ - радиус капли; $t$ - текущее время.

Уравнение (1) решалось при следующих начальных и граничных условиях:

$$
\begin{gathered}
t=0: T=T_{0}, 0 \leq r \leq R_{\mathrm{K}}, \\
r=0: \frac{\partial T}{\partial r}=0, \\
r=R_{\mathrm{K}}:-\lambda \frac{\partial T}{\partial r}=\alpha\left(T_{\mathrm{OC}}-T_{W}\right) .
\end{gathered}
$$

Здесь $T_{0}$ - начальная температура капли; $\alpha-$ коэффициент теплоотдачи; $T_{\text {ос }}$ - температура окружающего воздуха; $T_{W}$ - температура поверхности капли.

Область решения для второго варианта постановки задачи представлена на рис. 2.

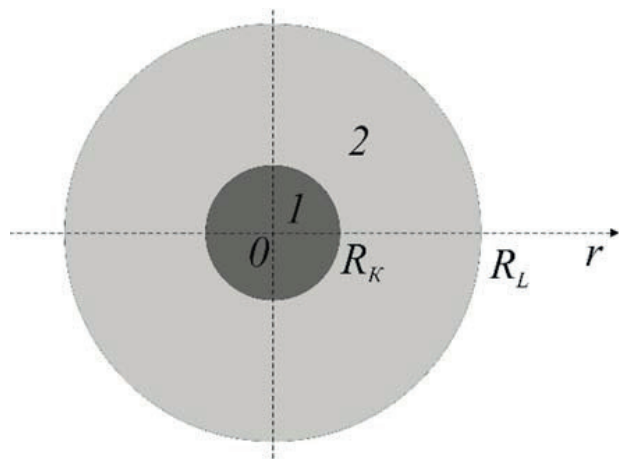

Pис. 2. Схема области решения для второго варианта постановки задачи

Fig. 2. Scheme of solution region for the second variant of the problem statement

Процесс тепломассопереноса для рассматриваемой постановки задачи описывается следующей системой дифференциальных уравнений в частных производных:

$$
\begin{gathered}
\rho c \frac{\partial T}{\partial t}=\lambda\left(\frac{\partial^{2} T}{\partial r^{2}}+\frac{2}{r} \frac{\partial T}{\partial r}\right), 0<r<R_{\mathrm{K}}, \\
\frac{\partial C_{\Pi}}{\partial t}=D\left(\frac{\partial^{2} C_{\Pi}}{\partial r^{2}}+\frac{2}{r} \frac{\partial C_{\Pi}}{\partial r}\right), R_{\mathrm{K}}<r<R_{L} .
\end{gathered}
$$

Здесь $C_{\Pi}, D$ - концентрация и коэффициент диффузии водяных паров соответственно. Остальные обозначения идентичны первому варианту постановки задачи.

Уравнения (2), (3) решались при следующих начальных и граничных условиях:

$$
\begin{gathered}
t=0: R_{\mathrm{K}}=R_{0}, \\
t=0: T=T_{0}, 0 \leq r \leq R_{\mathrm{K}}, \\
t=0: C_{\Pi}=\tilde{N}_{0}, R_{\mathrm{K}} \leq r \leq R_{L},
\end{gathered}
$$




$$
\begin{gathered}
r=0: \frac{\partial T}{\partial r}=0, \\
r=R_{\mathrm{K}}:-\lambda \frac{\partial T}{\partial r}=\alpha\left(T_{\mathrm{OC}}-T_{W}\right)+Q W . \\
r=R_{\mathrm{K}}:-D \frac{\partial C_{\Pi}}{\partial r}=\frac{W}{\rho} . \\
r=R_{L}: \frac{\partial C_{\Pi}}{\partial r}=0 .
\end{gathered}
$$

Здесь $R_{0}$ - радиус капли в начальный момент времени; $R_{L}$ - радиус расчетной области; $T_{W}-$ температура поверхности капли; $Q$ - теплота фазового перехода; $W$ - массовая скорость испарения воды, которая определялась по выражению [13]:

$$
W=\frac{A\left(P_{S}-P\right)}{\sqrt{2 \pi R T_{W} / M}},
$$

где $A$ - коэффициент аккомодации; $P_{S}$ - давление насыщенных паров воды; $P$ - парциальное давление паров вблизи границы испарения; $M$ - молекулярная масса воды; $T_{W}$ - температура поверхности. Вследствие относительно малых скоростей вдува водяных паров коэффициент аккомодации принят по данным [14].

Уравнение (1) и система уравнений (2), (3) с соответствующими начальными и граничными условиями решались методом конечных разностей с использованием четырехточечной неявной разностной схемы. Для решения нелинейных уравнений применялся метод итераций. Алгоритм решения задачи аналогичен использовавшемуся ранее при решении существенно нелинейных задач с комплексом термохимических превращений и несколькими подвижными границами [15]. Решение разностных аналогов дифференциальных уравнений и граничных условий проведено методом прогонки.

\section{Результаты и обсуждение}

Численное моделирование проведено при достаточно типичных значениях исходных данных: $R_{L}=0,1 \mathrm{~m} ; T_{0}=323 \mathrm{~K}, D=2,6 \cdot 10^{-5} \mathrm{M}^{2} / \mathrm{c}, C_{0}=0,01$. Было проанализировано два характерных значения температуры окружающего воздуха: 293 и $263 \mathrm{~K}$, соответствующих летнему и зимнему периодам года.

Так как решение нелинейной задачи с подвижной границей требует значительных вычислительных и временных ресурсов, то на первом этапе была проведена оценка влияния изменения радиуса капли при испарении на степень ее охлаждения. Для этого задача (2), (3) была решена с использованием двух подходов. В первом из них было учтено изменение радиуса капли во времени, обусловленное отводом водяных паров, образовавшихся на фронте испарения, в окружающую парогазовую смесь. При этом решение задачи с подвижной границей проводилось методом ловли фронта в узел пространственной сетки $[16,17]$. Во втором подходе изменение размера капли вследствие испарения не учитывалось.
На рис. 3 представлены зависимости относительного уменьшения радиуса капель вследствие их испарения с течением времени для различных начальных характерных размеров при $T_{\text {ос }}=293$ и $263 \mathrm{~K}$.
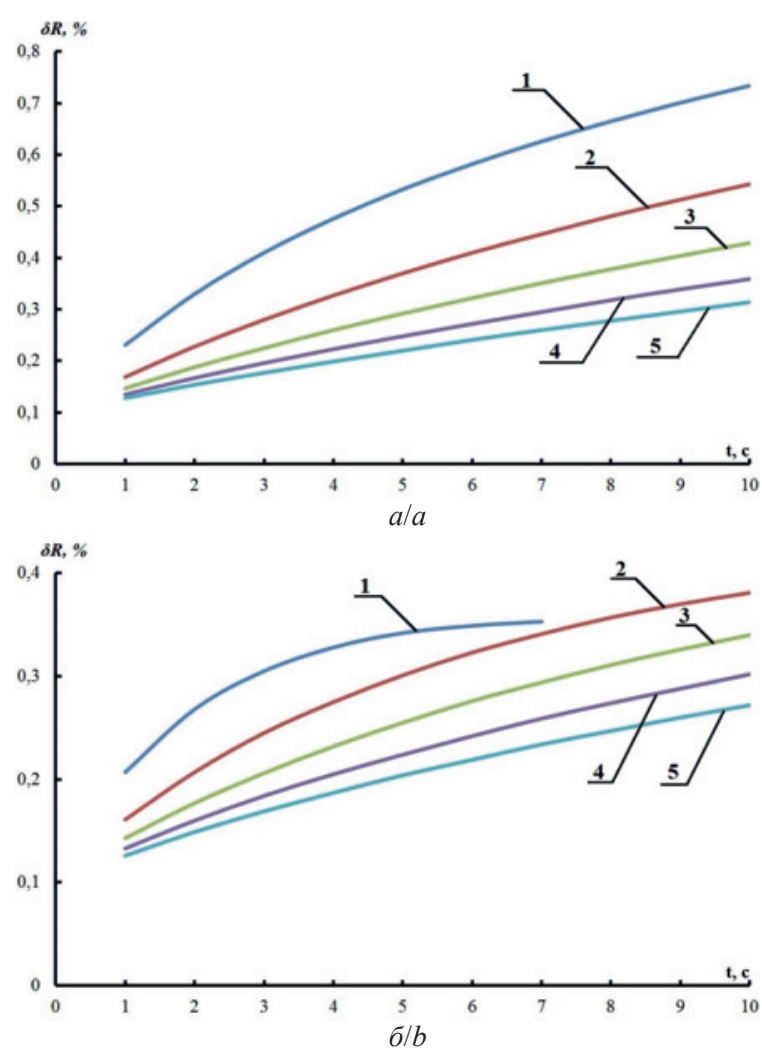

Pис. 3. Зависилость относительного уленьшения радиуса капли от времени при различных начальных размерах ( $T_{0 \mathrm{C}}=293 \mathrm{~K}$, б $\left.-T_{\mathrm{OC}}=263 \mathrm{~K}\right): R_{0}($ м. $): 1-1 ; 2-2 ; 3-3 ; 4-$ $4 ; 5-5$

Fig. 3. Time dependence of the relative decrease in the droplet radius for different initial sizes $\left(a-T_{0 \mathrm{C}}=293 \mathrm{~K}, b-T_{\mathrm{OC}}=263 \mathrm{~K}\right)$ : $R_{0}(m m): 1-1 ; 2-2 ; 3-3 ; 4-4 ; 5-5$

По результатам анализа зависимостей, представленных на рис. 3, можно сделать вывод о незначительном изменении размера капель за характерное время их полета при охлаждении воды в градирнях. При этом максимальное относительное изменение радиуса соответствует летнему периоду (рис. $3, a$ ) и через десять секунд полета составляет для капель, начальный радиус которых равен $1 \mathrm{mм}, 0,73 \%$, а для капель с $R_{0}=5 \mathrm{~mm}-0,31 \%$. На основании анализа зависимостей, представленных на рис. 3, б, можно отметить, что в зимний период для капли радиусом 1 мм через семь секунд полета испарение прекращается. Это обусловлено уменьшением давления насыщения ниже парциального давления водяных паров в пристеночной области. Это в свою очередь приводит к конденсации водяных паров на поверхности капли и росту ее температуры. Таким образом, можно сделать вывод о нецелесообразности чрезмерного дробления капель при отрицательных температурах наружного воздуха. 
Также проведен анализ влияния изменения характерного размера капель на охлаждение воды в градирнях. В таблице представлены значения среднеинтегральной температуры капель трех типичных размеров в характерные моменты времени, рассчитанные для различных начальных размеров с учетом $\left(T_{1}\right)$ и без учета $\left(T_{2}\right)$ изменения радиуса. На основании анализа значений, представленных в таблице, можно сделать вывод, что величина относительного расхождения при определении средней температуры капель $(\delta)$ максимальна в зимний период и в рассматриваемом диапазоне не превышает 0,06 \% .

Таблииа. Изменение среднеинтегральной телпературы ка пель во врелени с учетом изменения радиуса вслед ствие испарения и без него

Table. $\quad$ Change in average integral temperature of the droplets in time, taking into account the change in the radius due to evaporation and without it

\begin{tabular}{|c|c|c|c|c|c|c|c|c|c|}
\hline \multirow{4}{*}{$t, \mathrm{c}$} & \multicolumn{9}{|c|}{$T_{O C}=293 \mathrm{~K}$} \\
\hline & \multicolumn{9}{|c|}{$R_{0}(\mathrm{MM} / \mathrm{mm})$} \\
\hline & \multicolumn{3}{|c|}{1} & \multicolumn{3}{|c|}{3} & \multicolumn{3}{|c|}{5} \\
\hline & $T_{1}, \mathrm{~K}$ & $T_{2}, \mathrm{~K}$ & $\delta, \%$ & $T_{1}, \mathrm{~K}$ & $T_{2}, K$ & $\delta, \%$ & $T_{1}, \mathrm{~K}$ & $T_{2}, K$ & $\delta, \%$ \\
\hline 1 & 318,47 & 318,61 & 0,04 & 321,24 & 321,38 & 0,04 & 321,72 & 321,86 & 0,04 \\
\hline 5 & 309,14 & 309,30 & 0,05 & 317,58 & 317,72 & 0,04 & 319,45 & 319,58 & 0,04 \\
\hline 10 & 304,01 & 304,17 & 0,05 & 314,23 & 314,35 & 0,04 & 317,29 & 317,42 & 0,04 \\
\hline & \multicolumn{9}{|c|}{$T_{O C}=263 \mathrm{~K}$} \\
\hline 1 & 315,24 & 315,39 & 0,05 & 320,49 & 320,63 & 0,04 & 321,32 & 321,46 & 0,04 \\
\hline 5 & 297,09 & 297,28 & 0,06 & 314,90 & 315,03 & 0,04 & 318,08 & 318,21 & 0,04 \\
\hline 10 & - & - & - & 309,50 & 309,65 & 0,05 & 314,93 & 315,07 & 0,04 \\
\hline
\end{tabular}

Можно сделать вывод, что в условиях охлаждения, характерных для современных градирен, относительное изменение радиуса отдельных капель за характерное время полета не превышает $0,73 \%$, а максимальное относительное расхождение при определении их среднеинтегральной температуры при отсутствии учета изменения характерного размера вследствие испарения - 0,06 \% . Поэтому при дальнейшем анализе постановки (2), (3) изменение характерного размера капель не учитывалось.

На втором этапе проводился расчет распределения температуры по радиусу капли в предположении только конвективного охлаждения (задача (1)) и совместного испарительного и конвективного охлаждения (задача (2), (3)). Результаты представлены на рис. 4.

По результатам анализа зависимостей, представленных на рис. 4, можно сделать вывод о значительном вкладе испарительного охлаждения в распределение температуры по радиусу капли. При этом больший отвод теплоты за счет парообразования происходит в летний период. Так, при $T_{\text {ос }}=293 \mathrm{~K}$ через 1 секунду полета различие в температурах поверхности капли для двух рассматриваемых постановок составляет $2,5 \mathrm{~K}$, а через 10 секунд $-6,2 \mathrm{~K}$, а при $T_{\text {ос }}=263 \mathrm{~K}-2,2$ и $4,4 \mathrm{~K}$, соответственно.

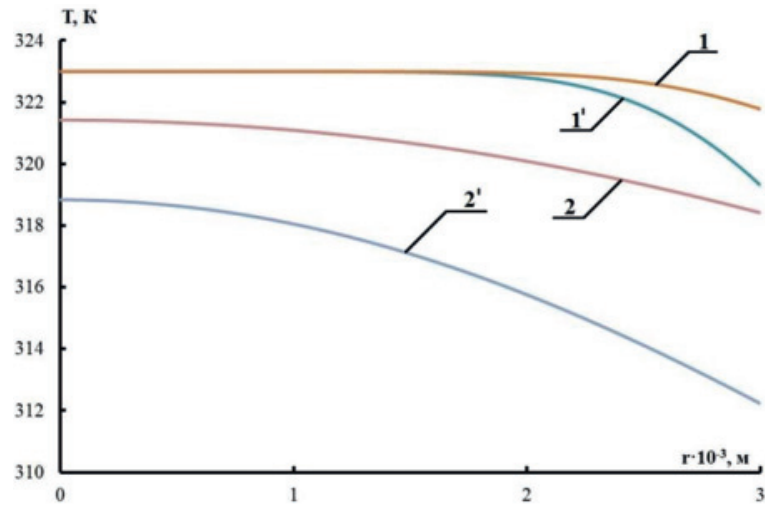

$a / a$

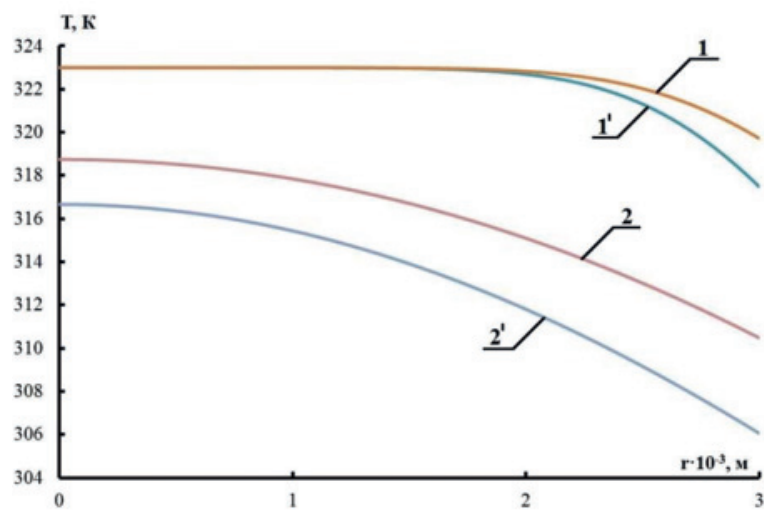

$\sigma / b$

Puc.4. Распределение телператур по радиусу капли $\left(R_{\mathrm{K}}=3\right.$ мл, $a-T_{\mathrm{OC}}=293 \mathrm{~K}$, б $\left.-T_{\mathrm{OC}}=263 \mathrm{~K}\right)$ в различные моменты времени: $1-t=1 c ; 2-t=10$ с (без индекса - без учета испарения, с индексом «" - с учетом)

Fig. 4. Distribution of temperatures along the radius of the drop $\left(R_{\mathrm{K}}=3 \mathrm{~mm}, a-T_{\mathrm{OC}}=293 \mathrm{~K}, b-T_{\mathrm{OC}}=263 \mathrm{~K}\right)$ at different time intervals: $1-t=1 \mathrm{~s} ; 2-t=10 \mathrm{~s}$ (without an index - without taking into account evaporation, with the index "'》-taking into account)

Значительное влияние на процессы тепломассопереноса оказывает характерный размер капель. На рис. 5 представлено распределение температур по радиусу для капель с характерным размером 1 и 5 мм через 1 и 10 секунд, в рамках двух рассматриваемых постановок.

На основании анализа зависимостей, представленных на рис. 5 , можно сделать вывод о большой роли испарительного охлаждения в формировании температурного поля капли. Так, температура поверхности капли, радиус которой равен 1 мм, при $T_{\text {ос }}=293 \mathrm{~K}$, вычисленная в рамках двух рассматриваемых постановок, отличается на $2,8 \mathrm{~K}$ и $5,7 \mathrm{~K}$ через 1 и 10 с полета соответственно.

На рис. 6 представлены среднеинтегральные температуры капель трех типичных размеров в характерные моменты времени, вычисленные с учетом испарительного охлаждения и без учета.

На основании анализа представленных зависимостей можно сделать вывод, что с течением времени разность температур, соответствующих только конвективному и конвективно-испарительному 
охлаждению, растет. При этом большую роль испарительное охлаждение играет в летний период. Так, для капли радиусом 3 мм через десять секунд полета разница среднеинтегральных температур капель для постановки задачи с учетом испарения и без него составила: при $T_{0 с}=293 \mathrm{~K}-5,0 \mathrm{~K}$, а при $T_{\text {oC }}=263 \mathrm{~K}-3,7 \mathrm{~K}$.
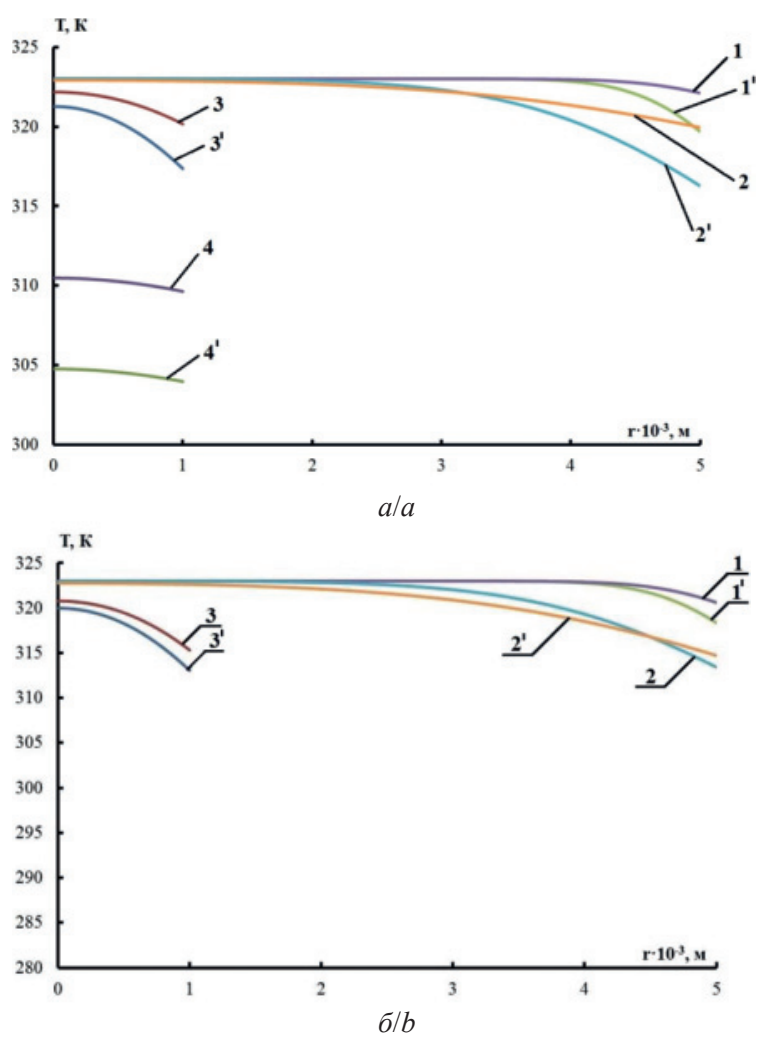

Puс. 5. Распределение температуры по радиусу капли при раз личных начальных размерах через 1 и 10 секунд полета $\left(a-T_{\mathrm{OC}}=293 \mathrm{~K}\right.$, б $\left.-T_{\mathrm{OC}}=263 \mathrm{~K}\right): 1,2-R_{\mathrm{K}}=5 \mathrm{m.л}, 3,4-$ $R_{\mathrm{K}}=1$ мм (без индекса - без учета испарения, $с$ индексом «'»-с учетол).

Fig. 5. Temperature distribution along the radius of the drop for different initial sizes after 1 and 10 seconds of flight ( $a$ $\left.T_{\mathrm{OC}}=293 \mathrm{~K}, b-T_{\mathrm{OC}}=263 \mathrm{~K}\right): 1,2-R_{\mathrm{K}}=5 \mathrm{~mm}, 3,4-R_{\mathrm{K}}=1 \mathrm{~mm}$ (without an index - without taking into account evaporation, with the index «"》-taking into account)

Среднеинтегральные температуры капель составили: при $R_{\mathrm{K}}=3$ мм через 2 секунды полета 320,3 и 322,0 K, через 7 с полета 316,3 и 320,5 К. Для $T_{\text {ос }}=263 \mathrm{~K}$ через 2 с эти величины составили соответственно 319,3 ; 320,5 ; а через 7 с 312,7 ; 315,8 К. Следовательно, можно сделать вывод, что поглощение теплоты испарением при охлаждении капель воды в градирнях играет важную роль в формировании температурных полей капель охлаждаемой воды на выходе из градирен. Полученные результаты также показывают, что повышение әффективности охлаждения оборотной воды в градирнях возможно при анализе процессов тепломассопереноса как в каплях воды, так и в их малой окрестности с использованием математического аппарата, разработанного в последние годы [18], эффективно применяющегося при решении группы задач топливных [19] и химических [20] технологий с использованием капельных потоков.
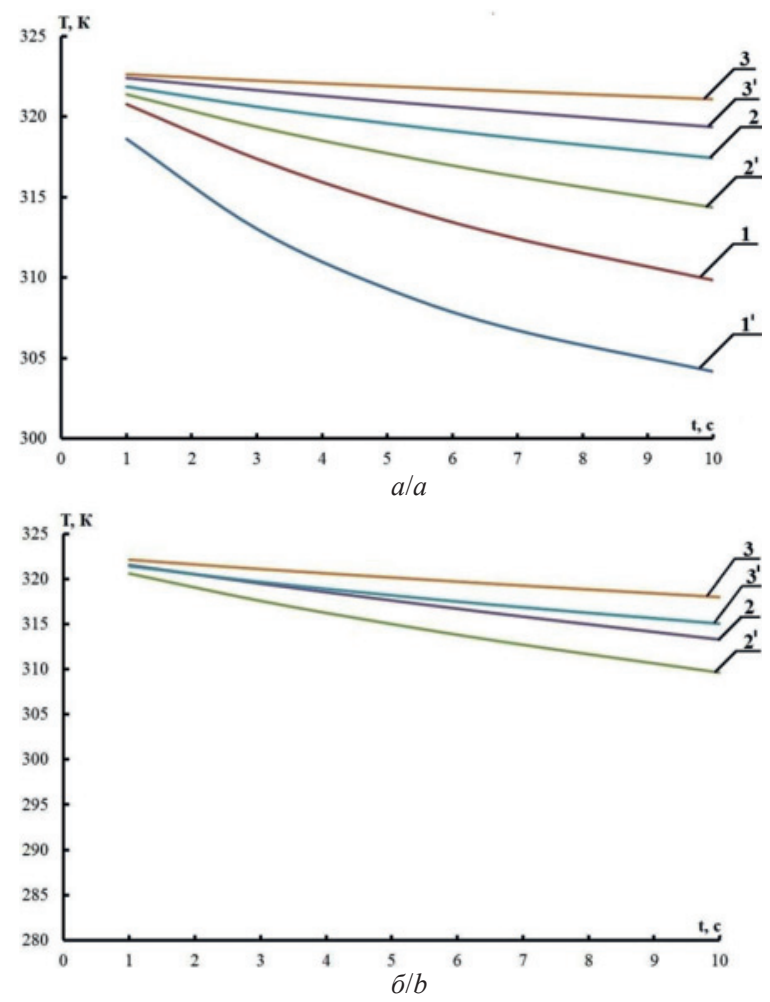

Pис.6. Зависилость среднеинтегральных телператур капли от времени при различных начальных радиусах, вычисленные с использованием двух рассматриваемых постановок $\left(a-T_{\text {ос }}=293 \mathrm{~K}\right.$, б $\left.-T_{\mathrm{OC}}=263 \mathrm{~K}\right): R_{\mathrm{K}}($ м. $): 1-1 ; 2-3$; 3 - 5 (без индекса» - без учета испарения, с индексом «"»-с учетол).

Fig. 6. Time dependence of drop average integral temperatures on different initial radii, calculated using two considered sets $\left(a-T_{\mathrm{OC}}=293 \mathrm{~K}, b-T_{\mathrm{OC}}=263 \mathrm{~K}\right): R_{\mathrm{K}}(\mathrm{mm}): 1-1 ; 2-3 ; 3-5$ (without an index - without taking into account evaporation, with the index «'»-taking into account)

\section{Заключение}

На основании результатов решения задачи о температурном поле капли оборотной воды в достаточно типичных условиях работы градирен установлено, что, несмотря на незначительные изменения радиусов капель (не более $0,73 \%$ за время их полета), испарение воды играет значительную роль в формировании температурного поля капель любых размеров. При этом большую роль отвод теплоты за счет парообразования играет в летний период года.

Показано, что в зимний период существует предельное минимальное значение радиуса капель, зависящее от температуры окружающего воздуха, при котором процесс испарительного охлаждения прекращается. 


\section{СПИСОК ЛИТЕРАТУРЫ}

1. Alavi S.R., Rahmati M. Experimental investigation on thermal performance of natural draft wet cooling towers employing an innovative wind-creator setup // Energy Conversion and Management. - 2016. - V. 122. - P. 504-514.

2. Numerical study of water flow rates in power plant cooling systems / P. Regucki, M. Lewkowicz, R. Krzyżyńska, H. Jouhara // Thermal Science and Engineering Progress. - 2018. - V. 7. P. 27-32.

3. Performance recovery of natural draft dry cooling systems by combined air leading strategies / W. Wang, L. Chen, X. Huang, L. Yang, X. Du. // Energies. - 2017. - V. 10. - № 12. - P. 1-18.

4. Sellamuthu P., Manoharan C., Senthil Kumar R. Theoretical analysis of a wet cooling tower coupled with a desalination plant for fresh water yield / Desalination and Water Treatment. - 2017. V. 93. - P. 1-8.

5. Roux D., Reuter H.C.R. Performance evaluation and design of a new cooling tower spray system for uniform water distribution // Heat Transfer Engineering. - 2017. - V. 38. - № 11-12. P. 1044-1053.

6. Krahé D., Beisheim B., Engell S. Decision support for energy-efficient cooling tower operation using weather forecasts // Chemical Engineering Transactions. - 2016. - V. 52. - P. 1009-1014.

7. Испарительное охлаждение капель жидкости в воздушном потоке / Н.А. Меренцов, В.А. Балашов, А.Б. Голованчиков, Я.А. Орлянкина // Известия Волгоградского государственного технического университета. - 2012. - Т. 1. - № 5. C. $78-80$.

8. Экспериментальное и численное исследования нестационарного испарения капель жидкости / В.И. Терехов, В.В. Терехов, Н.Е Шишкин, К.Ч. Би // Инженерно-физический журнал. 2010. - T. 83. - № 5. - C. 829-836.

9. Терехов В.И., Шишкин Н.Е. Испарение капель воды с углеродными нанотрубками // Письма в журнал технической физики. - 2012. - Т. 38. - № 1. - С. 51-57.

10. Терехов В.И., Шишкин Н.Е. Адиабатическое испарение бинарных смесей жидкости на поверхности пористого шара // Теплофизика и аэромеханика. - 2009. - Т. 16. - № 2. C. $253-259$.
11. Волков Р.С., Кузнецов Г.В, Стрижак П.А. Влияние начальных параметров распыленной воды на характеристики ее движения через встречный поток высокотемпературных газов // Журнал технической физики. - 2014. - Т. 84. - № 7. C. $15-23$.

12. Кузнецов Г.В., Стрижак П.А. Движение совокупности капель мелкодисперсной жидкости во встречном потоке высокотемпературных газов // Письма в журнал технической физики. 2014. - T. 40. - № 12. - C. 11-18.

13. Полежаев Ю.В., Юрьевич Ф.Б. Тепловая защита. - М.: Энергия, 1976. - 392 с.

14. Кузнецов Г.В., Куйбин П.А., Стрижак П.А Оценка численных значений констант испарения капель воды, движущихся в потоке высокотемпературных газов // Теплофизика высоких температур. - 2015. - Т. 53. - № 2. - С. 264-269.

15. Математическое моделирование теплофизических и термохимических процессов при горении вспучивающихся огнезащитных покрытий / В.П. Страхов, А.Н. Гаращенко, Г.В. Кузнецов, В.П. Рудзинский // Физика горения и взрыва. - 2001. T. 37. - № 2. - C. 63-73.

16. Сыродой С.В., Кузнецов Г.В., Саломатов В.В. Влияние условий теплообмена на характеристики зажигания частиц водоугольного топлива // Теплоэнергетика. - 2015. - № 10. C. $16-21$.

17. Кузнецов Г.В., Саломатов В.В., Сыродой С.В. Численное моделирование зажигания частиц водоугольного топлива // Физика горения и взрыва. - 2015. - Т. 51. - № 4. - С. 11-19.

18. Sazhin S.S., Shishkova I.N., Al Qubeissi M. A self-consistent kinetic model for droplet heating and evaporation // International Journal of Heat and Mass Transfer. - 2016. - V. 93. P. 1206-1217.

19. Sazhin S.S. Modelling of fuel droplet heating and evaporation: Recent results and unsolved problems // Fuel. - 2017. - V. 196. P. 69-101.

20. Sazhin S.S., Gun'ko V.M., Nasiri R. Quantum-chemical analysis of the processes at the surfaces of Diesel fuel droplets // Fuel. 2016. - V. 165. - P. 405-412.

Поступила 13.09.2018 2.

\section{Информация об авторах}

Шевелев C.A., старший преподаватель научно-образовательного центра И.Н. Бутакова Национального исследовательского Томского политехнического университета.

зяблова $\boldsymbol{H . H . , ~ д о ц е н т ~ о т д е л е н и я ~ и н о с т р а н н ы х ~ я з ы к о в ~ ш к о л ы ~ б а з о в о и ̆ ~ и н ж е н е р н о и ̆ ~ п о д г о т о в к и ~ Н а ц и о н а л ь н о - ~}$ го исследовательского Томского политехнического университета. 
UDC 621.175

\title{
ON THE EFFECT OF WATER EVAPORATION ON COOLING EFFICIENCY IN COOLING TOWERS
}

\author{
Sergey A. Shevelev', \\ wevelev@tpu.ru \\ Natalia N. Zyablova', \\ zyablova@tpu.ru \\ 1 National Research Tomsk Polytechnic University, \\ 30, Lenin Avenue, Tomsk, 634050, Russia.
}

\begin{abstract}
The paper introduces the results of theoretical study of cooling water droplets as they move in a stream of cold air under conditions characteristic of high-capacity tower cooling towers and the results of a numerical analysis of temperature distributions along the droplet radius. Due to the large temperature differences and significant speeds in the system under consideration, the temperature distribution inside the droplets is assumed to vary along the radius. The increase in air humidity in the near-wall area due to water evaporation is taken into account. The resulting system of nonstationary partial differential equations is solved by the finite difference method using an implicit four-point difference scheme. The solution of the problem with a moving boundary was carried out by the method of catching the front into a node of a spatial grid. To solve nonlinear equations, the iteration method was used. The aim of the work is a numerical evaluation of convective and evaporative cooling contribution to formation of the temperature field of water droplets under conditions typical of modern cooling towers. The analysis was carried out for two characteristic values of the ambient air temperature corresponding to the summer and winter periods of the year. The analysis of the effect of cooling droplets due to water evaporation and thermal conductivity was performed. The temperature distribution over the radius is obtained for different values of the characteristic sizes of droplets. It is shown that the change in radius for the entire range of characteristic sizes of droplets during their flight is less than 0,73\% and does not have a significant effect on the amount of water cooling in the cooling tower. It is established that the absorption of heat by evaporation plays an important role in formation of temperature distribution in the droplets of circulating water at the outlet of the cooling towers. It is shown that at negative ambient temperatures, excessive crushing of droplets is impractical.
\end{abstract}

Key words:

Recycling water, heat and mass transfer, cooling tower, water evaporation, convection.

\section{REFERENCES}

1. Alavi S.R., Rahmati M. Experimental investigation on thermal performance of natural draft wet cooling towers employing an innovative wind-creator setup. Energy Conversion and Management, 2016, vol. 122, pp. 504-514.

2. Regucki P., Lewkowicz M., Krzyżyńska R., Jouhara H. Numerical study of water flow rates in power plant cooling systems. Thermal Science and Engineering Progress, 2018, vol. 7, pp. 27-32.

3. Wang W., Chen L., Huang X., Yang L., Du X. Performance recovery of natural draft dry cooling systems by combined air leading strategies. Energies, 2017, vol. 10, no. 12, pp. 1-18.

4. Sellamuthu P., Manoharan C., Senthil Kumar R. Theoretical analysis of a wet cooling tower coupled with a desalination plant for fresh water yield. Desalination and Water Treatment, 2017, vol. 93 , pp. 1-8.

5. Roux D., Reuter H.C.R. Performance evaluation and design of a new cooling tower spray system for uniform water distribution. Heat Transfer Engineering, 2017, vol. 38, no. 11-12, pp. 1044-1053.

6. Krahé D., Beisheim B., Engell S. Decision support for energy-efficient cooling tower operation using weather forecasts. Chemical Engineering Transactions, 2016, vol. 52, pp. 1009-1014.

7. Merencov N.A., Balashov V.A., Golovanchikov A.B., Orlyankina Ya.A. Isparitelnoe okhlazhdenie kapel zhidkosti v vozdushnom potoke [Evaporation cooling of liquid drops in air flow]. Izvestiya Volgogradskogo gosudarstvennogo tekhnicheskogo universiteta, 2012, vol. 1, no. 5, pp. 78-80.

8. Terekhov V.I., Terekhov V.V., Shishkin N.E., Bi K.Ch. Heat and mass transfer in disperse and porous media experimental and numerical investigations of nonstationary evaporation of liquid droplets. Journal of Engineering Physics and Thermophysics, 2010, vol. 83 , no. 5. pp. 883-890.
9. Terekhov V.I., Shishkin N.E. Evaporation of water droplets containing carbon nanotubes. Technical Physics Letters, 2012, vol. 38 , no. 1, pp. 25-28.

10. Terekhov V.I., Shishkin N.E. Adiabatic evaporation of binary liquid mixtures on the porous ball surface. Thermophysics and Aeromechanics, 2009, vol. 16, no. 2, pp. 239-245.

11. Volkov R.S., Kuznetsov G.V., Stizhak P.A. Influence of the initial parameters of spray water on its motion through a counter flow of high-temperature gases. Technical Physics, 2014, vol. 59, no. 7, pp. 959-967.

12. Kuznetsov G.V., Stizhak P.A. The motion of a manifold of finely dispersed liquid droplets in the counterflow of high-temperature gases. Technical Physics Letters, 2014, vol. 40, no. 6, pp. 499-502.

13. Polezhaev Ju.V., Yurevich F.B. Teplovaya zashchita [Thermal shield]. Moscow, Energiya Publ., 1976. 391 p.

14. Kuznetsov G.V., Kuybin P.A., Stizhak P.A. Estimation of the numerical values of the evaporation constants of water droplets moving in a high-temperature gas flow. High Temperature, 2015, vol. 53 , no. 2, pp. 254-258.

15. Strakhov V.L., Garashchenko A.N., Kuznetsov G.V., Rudzinskii V.P. Mathematical simulation of thermophysical and thermochemical processes during combustion of intumescent fire-protective coatings. Combustion explosion and shock waves, 2001, vol. 37, no. 2, pp. 178-186.

16. Syrodoy S.V., Kuznetsov G.V., Salomatov V.V. The influence of heat transfer conditions on the parameters characterizing the ignition of coal-water fuel particles. Thermal Engineering, 2015, vol. 62, no. 10, pp. 703-707.

17. Kuznetsov G.V., Syrodoy S.V., Salomatov V.V. Numerical simulation of ignition of particles of a coal-water fuel. Combustion, Explosion, and Shock Waves, 2015, vol. 51, no. 4, pp. 409-415. 
18. Sazhin S.S., Shishkova I.N., Al Qubeissi M. A self-consistent kinetic model for droplet heating and evaporation. International Journal of Heat and Mass Transfer, 2016, vol. 93, pp. 1206-1217.

19. Sazhin S.S. Modelling of fuel droplet heating and evaporation: Recent results and unsolved problems. Fuel, 2017, vol. 196, pp. $69-101$.

\section{Information about the authors}

Sergey A. Shevelev, senior lecturer, National Research Tomsk Polytechnic University.

Natalia N. Zyablova, assistant professor, National Research Tomsk Polytechnic University.
20. Sazhin S.S., Gun'ko V.M., Nasiri R. Quantum-chemical analysis of the processes at the surfaces of Diesel fuel droplets. Fuel, 2016, vol. 165 , pp. $405-412$.

Received: 13 September 2018. 